Henning Wilts, Nadja von Gries

\title{
Suffizienz als \\ Geschäftsfeld der \\ Kreislaufwirtschaft
}

Ursprünglich veröffentlicht in:

Umweltwirtschaftsforum, 23 (2015), 1, 41-47

DOI: $10.1007 /$ soo550-015-0351-x 
Henning Wilts $a$, *

Nadja von Gries a

\section{Suffizienz als Geschäftsfeld der Kreislaufwirtschaft}

a Wuppertal Institut für Klima, Umwelt, Energie, Wuppertal

* Korrespondierender Autor:

Henning Wilts

Wuppertal Institut für Klima, Umwelt, Energie

Döppersberg 19

42103 Wuppertal

Deutschland

E-Mail: henning.wilts@wupperinst.org

Tel.: +49 202 2492-139

Fax: +49 202 2492-108

Dies ist die begutachtete, akzeptierte Manuskriptversion des Artikels. Aus dem Veröffentlichungsprozess können nachträgliche Änderungen resultieren, die sich z. B. auf die Formatierung und Zeichensetzung auswirken. Derartige Änderungen sind hier nicht berücksichtigt. Die endgültige Version wurde in der oben genannten Zeitschrift veröffentlicht. 


\section{Einleitung}

Die in der abfallwirtschaftlichen Diskussion befindlichen Strategien zur Entlastung der Ökosysteme fokussieren bislang überwiegend auf technische Effizienz- und Konsistenzstrategien. Insbesondere aufseiten der Wirtschaft soll der Gesamtressourcenverbrauch mit Hilfe technologischen Fortschritts und geschlossener Stoffkreisläufe gesenkt werden. Daneben beschreibt die Literatur sogenannte Suffizienzstrategien, welche nicht allein eine relative Senkung des Ressourcenverbrauchs, sondern mit Hilfe von Strategien eines „Weniger“, "Langsamer", „Regionaler" eine absolute Ressourcensenkung anstreben (Schneidewind und Palzkill 2012; Sachs 1993). Suffizienzstrategien werden jedoch, wenn überhaupt, häufig allein auf Seiten individueller Konsumentscheidung diskutiert (vgl. Stengel 2011; Linz et al. 2002; Linz 2012; Paech 2012). Insbesondere angesichts weiter steigender globaler Gesamtressourcenverbräuche (Bringezu und Bleischwitz 2009) ist dies erstaunlich und lenkt den Blick auf Suffizienzstrategien, welche über die Ebene der individuellen Konsumentscheidungen hinausgehen und die Potentiale unternehmerischer Suffizienzstrategien in den Blick nehmen.

Ein in diesem Zusammenhang interessantes Fallbeispiel bildet der Transitionsprozess der Abfallwirtschaft, deren Funktion historisch auf die Gewährleistung einer Entsorgungssicherheit ausgelegt war - Abfälle sollten zuverlässig, billig und seit den 1960er Jahren möglichst auch umweltfreundlich entsorgt werden. Aktuell entwickeln sich jedoch neue Geschäftsmodelle, die über reine End-of-Pipe Technologien hinausgehen und auf ein nachhaltiges Ressourcenmanagement abzielen. Interessanterweise spielen dabei auch Suffizienzaspekte eine immer wesentlichere Rolle, weil sowohl Effizienz- und Konsistenz-Ansätze in vielen Bereichen absehbar an ihre Grenzen stoßen. Im Folgenden soll diese Rolle suffizienter Geschäftsmodelle in der Abfallwirtschaft aufgezeigt und anhand konkreter Fallbeispiele diskutiert werden. Daraus lassen sich Schlussfolgerungen zur Rolle technischer Pfadabhängigkeiten, zur Messbarkeit von Suffizenz und zur Integration von Suffizienzaspekten in der Infrastrukturplanung ziehen, die über abfallwirtschaftliche Geschäftsmodelle hinausgehen.

\section{Transitionsprozesse in der Abfallwirtschaft}

Historisch ist die Abfallwirtschaft als Geschäftsfeld ein Stadtphänomen: Erst durch die Bildung von städtischen Siedlungsgemeinschaften entstanden unerwünschte Stoffströme wie Exkremente und Kadaver in einer Menge, dass damit vor allem gesundheitsgefährdende hygienische Probleme für den Menschen verbunden waren. Ohne diese Systeme konnten Siedlungen eine bestimmte Größe nur überschreiten, wenn sie z. B. unmittelbar an Flüssen lagen, mit denen Abfälle entsorgt werden konnten. Mit Beginn der Industrialisierung wurde Abfall jedoch $\mathrm{zu}$ einem „allgegenwärtigem Unwertphänomen“ (Kuchenbuch 1989, S. 257). Der Übergang von der Abfallentsorgung zur Abfallbewirtschaftung in Deutschland war weniger durch direkte Umweltschäden der Abfallerzeugung, sondern vor allem durch zunehmende Knappheiten an Entsorgungskapazitäten, verursacht durch das dem Wirtschaftswunder folgende „Abfallwunder“ (Lamping 1998, S. 63) geprägt: Die rasant steigenden Konsumausgaben und die zunehmende Verbreitung vor allem von Plastikverpackungen führten sehr schnell zu Engpässen bei der Deponie- und Verbrennungskapazität; ein wichtiger Faktor war dabei auch die Umstellung von der häuslichen Lebensmittelverarbeitung zu industriell vorgefertigten Nahrungsmitteln (Wollny 1992, S. 14). Die Abfall-Politik in Deutschland reagierte Anfang der 1990er 
Jahre auf den sich abzeichnenden Entsorgungsnotstand und die stetig steigenden Entsorgungskosten mit einer Zäsur des Abfallrechts: Verwertbare Abfälle wurden durch das KrW-/AbfG zu (zumindest im Inland) frei handelbaren Wirtschaftsgütern erklärt (Hemmer et al. 2003, S. 11). In Zukunft sollte der Markt durch den Ausgleich von Angebot und Nachfrage für die ökonomisch effizienteste Verteilung dieser Güter auf die verschiedenen technischen Verwertungswege sorgen. Prinzipiell sollte der Abfallerzeuger zuständig für die Nachnutzungsphase der von ihm in Verkehr gebrachten Produkte sein, solange es sich um verwertbare Abfälle handelt. Diese Umsetzung des Verursacherprinzips sollte den Einstieg in eine ökologische Stoffflusswirtschaft durch eine wirtschaftlich getriebene Verwertung ermöglichen (Schenkel 2003). Damit wurde jedoch gleichzeitig der Startschuss für die Entstehung einer privaten Abfallwirtschaft im Bereich der Haushaltsabfälle gegeben: Da Abfälle im gesellschaftlichen Umweltverständnis zunehmend $\mathrm{zu}$ wertvollen Sekundärrohstoffen wurden, sollten private Unternehmen sich ihrer annehmen und so den Staat in seiner Zuständigkeit für die Entsorgung entlasten. Gleichzeitig entwickelte sich damit aber auch ein renditeorientierter Industriezweig, der von einem konstant hohen Abfallaufkommen abhing.

Die aktuelle Diskussion um die Transformation zu einer Kreislaufwirtschaft hat insbesondere auf europäischer Ebene durch die Aussetzung des zuvor mit Spannung erwarteten sogenannten „Circular Economy Package“ durch die neue EUKommission erheblich an Aufmerksamkeit gewonnen. Der vorgelegte Entwurf wurde entweder als $\mathrm{zu}$ ambitioniert oder $\mathrm{zu}$ technokratisch kritisiert (vgl. Wilts und Schepelmann 2015); aber auch das massiv von der Ellen MacArthur Foundation propagierte Konzept eines geschlossenen Wirtschaftssystems (vgl. Abb. 1) offenbart noch erhebliche konzeptionelle Schwachstellen. 


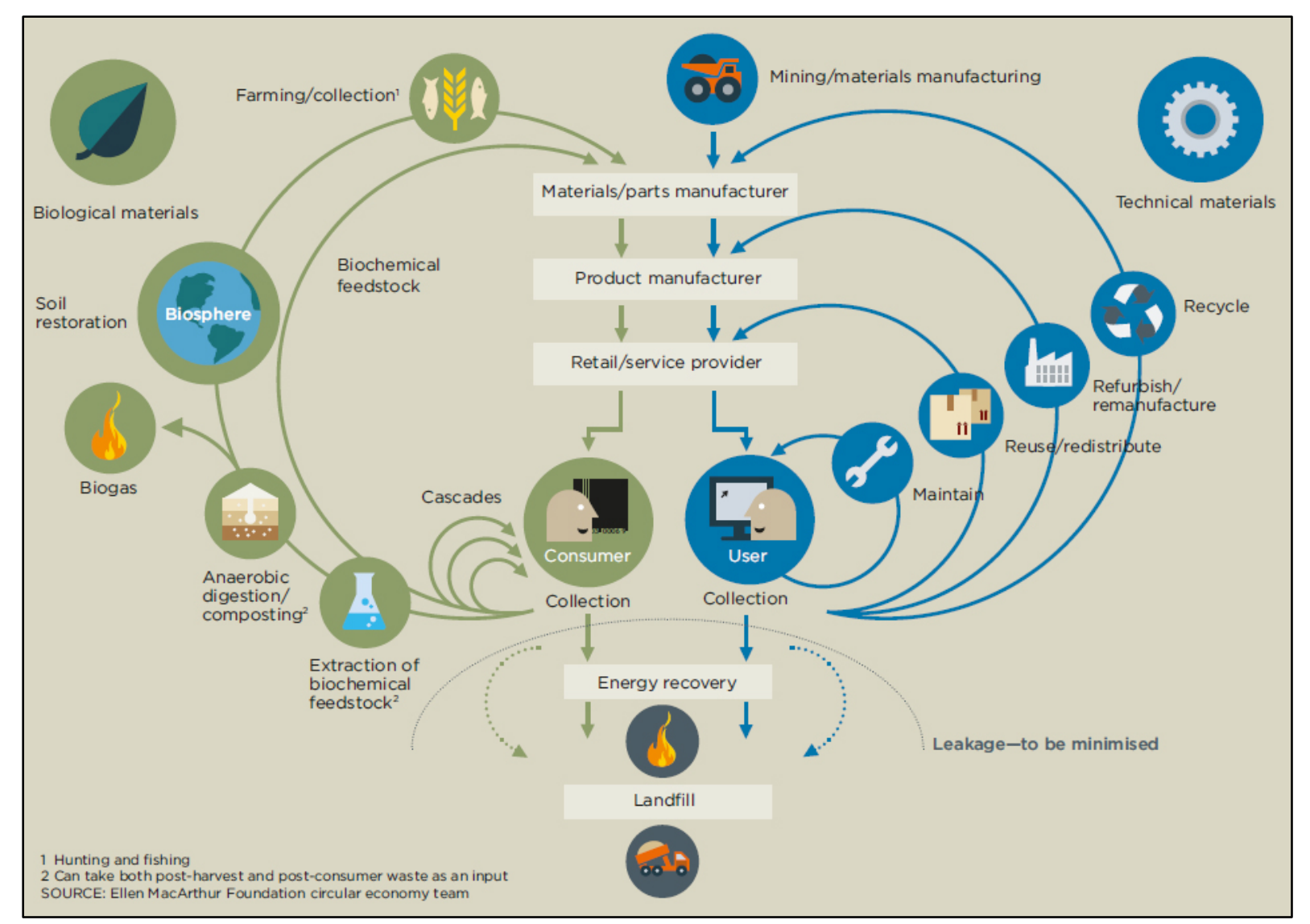

Abbildung 1: Geschlossene Stoffkreisläufe in der Kreislaufwirtschaft Quelle: EMF 2013, Report 2

- Das Konzept ist offensichtlich inspiriert von den Vorstellungen eines Cradleto-Cradle Designs (u. a. die Aufteilung in einen „technischen" und einen „biologischen“ Kreislauf, vgl. dazu McDonough und Braungart 2010) und folgt damit einem klaren Konsistenzansatz, die Entstehung von Abfällen durch Änderungen des Produktdesigns unschädlich zu gestalten. Damit steht das Konzept jedoch auch im klaren Widerspruch zur Abfallhierarchie, wie sie u. a. in der Europäischen Abfallrahmenrichtlinie verankert ist: Demnach ist die Vermeidung von Abfällen der Schließung von Stoffkreisläufen durch Recycling grundsätzlich überlegen und bei der Formulierung von Abfallpolitiken und Planungsprozessen prioritär zu verfolgen. Um im Bild zu bleiben: Es geht nicht nur um das Schließen der Stoffkreisläufe, sondern um ihre Verkleinerung. Auffällig ist dass hier weder die notwendige Energie zur Schließung von Stoffkreisläufen und ihre Auswirkungen auf das Klima, noch die unvermeidlichen Materialverluste in Recyclingprozessen oder das Thema Kritikalität von Rohstoffen auch nur erwähnt werden.

- Aber auch stärker effizienzorientierte Ansätze werden der Herausforderung einer Transformation in Richtung Kreislaufwirtschaft nur in Teilen gerecht, wenn sie auf die Ressourceneinsparpotentiale durch den Einsatz von Sekundärrohstoffen verweisen. Zum einen werden hier die komplexen sozioökonomischen Zusammenhänge weitestgehend vernachlässigt, die zur Entstehung von Abfällen, ihrer Sammlung und Behandlung und den damit verbundenen Umweltbelastungen führen. Zum anderen lauert hier auch schnell die Gefahr klassischer Rebound-Effekte (Sachs 2002, S. 52): Da Sekundärrohstoffe häufig zu geringeren Kosten eingesetzt werden können als 
Primärmaterialien, erhöht sich im Endeffekt der Stoffdurchsatz und die erreichten Umweltvorteile werden zumindest teilweise kompensiert.

Befördert durch diese konzeptionellen Lücken geraten damit zunehmend auch wieder Suffizienzstrategien in den Fokus der Abfallwirtschaft - insbesondere wenn sie z. B. im Rahmen einer sich abzeichnenden Entwicklung zur Sharing- oder Leasing Economy (vgl. Fischer et al. 2015) Optionen für innovative Geschäftsmodelle bieten. Suffizienzstrategien als stark normativ geprägtes Konzept fokussieren traditionell auf das rechte Maß des Konsums: „Es fordert nicht, auf das Notwendige zu verzichten, sondern mit dem Ausreichenden zufrieden zu sein." (Scherhorn 2002, S. 16) Damit wird das grundsätzliche Spannungsverhältnis zwischen Suffizienz und Geschäftsmodellen deutlich (Wilts und Palzkill 2014).

\section{Konkrete Fallbeispiele suffizienzorientierter Geschäftsmodelle in der Abfallwirtschaft}

Mit Bezug auf die Entstehung von Abfällen ist die Suffizienzperspektive aber durchaus relevant, weil sie die Notwendigkeit von Konsumprozessen als Auslöser von Ressourcenverbräuchen und anschließender Entstehung von Abfällen hinterfragt und damit auch Kosteneinsparpotenziale bietet. Im Folgenden sollen zwei solcher Ansätze näher beleuchtet werden: Die Übertragung des Contracting-Modells auf den Abfallbereich und die Wiederentdeckung des Reparierens als Gesellschaftsphänomen.

\section{Fallbeispiel Abfall-Contracting}

Ein möglicher Ansatz solcher ressourcenleichten Geschäftsmodelle, die bestehende Nutzenaspekte mit veränderten Konsummustern und niedrigerem Ressourcenverbrauch bereitstellen, sind Contracting-Modelle, die bisher vor allem im Energiesektor entwickelt wurden: Sie gehören zu den wenigen Geschäftsmodellen, welche ein „Weniger“ zu einem etablierten Business Case zu machen. Contracting gilt als eine klassische Finanzierungsstrategie von Effizienzmaßnamen zur Energieeinsparung. Es lässt sich jedoch auch als Suffizienzstrategie fassen, da sich hierdurch sowohl auf Seiten der Gebäudeeigentümer als als auch beim Mieter die Strukturen der Bedürfnisbefriedigung durch Energieversorgung und -nutzung verändern. Folgt man einer auf Nutzenaspekte abzielenden Definition von Suffizienz, so fördert das Contracting einen Perspektivenwechsel von „Heizen“ zu „Wohnkomfort“ - wobei in diesem Fall eine effiziente Dienstleistung in Anspruch genommen wird. Dieser Dienstleister profitiert an der realen Senkung des absoluten Energieverbrauchs, womit (direkte) Rebound-Effekte verhindert werden können (Stengel 2011; Sommer 2012).

Die Übertragung des Prinzips des Performance-Contractings vom Energie- auf den Abfallsektor war Ausgangspunkt u. a. des 1996 in Kiel gegründeten Unternehmens Innotec. Die Innovation zielt auf ein „finanzielles Anreizmodell“ zur verbesserten Abfalltrennung durch die Mieter und damit nachhaltige Kostensenkungen bei der „Bewirtschaftungen von Wohnimmobilien“ (Innotec Abfallmanagement o. J.). Das Geschäftsmodell basiert auf zwei unterschiedlichen Säulen: Der Bereich „Konventionelles Abfallmanagement“ besteht aus einer intensiven persönlichen Beratung der Mieter zur korrekten Abfalltrennung und zur Vermeidung von Abfällen in verschiedenen Sprachen mit zielgruppenspezifischen Kampagnen wie „Platz ist in der kleinsten Küche“. Der Schwerpunkt der Tätigkeiten liegt jedoch auf der mehrmals 
in der Woche stattfindenden Sichtprüfung der Restmüllgefäße durch Personal von Innotec, den sogenannten Liegenschaftsbetreuern, bei der Sperrmüll und großvolumiger Abfall wie Pappkartons oder andere Großverpackungen aussortiert werden, um somit eine Reduzierung der Restmüllmengen zu erreichen. Diese Aufgabe muss auch nach intensiver Beratung immer wieder und regelmäßig durchgeführt werden, über die Beratung allein sind zumindest keine dauerhaften Veränderungen des Mülltrennungsverhaltens zu erreichen. Innotec betont dabei, dass es sich aus abfallrechtlicher Sicht nicht um eine Sortierung der Abfälle im technischen Sinne, sondern lediglich um eine Behebung von Befüllungsfehlern handelt. Es findet dabei weder eine Entnahme von Wertstoffen - alle überlassungspflichtigen Abfälle zur Entsorgung werden auch tatsächlich dem öffentlich- rechtlichen Entsorgungsträger überlassen - noch eine Verpressung von Abfällen statt. Daneben gewährleistet das Unternehmen auch eine regelmäßige Reinigung der Standplätze. Darüber hinaus bietet Innotec als Weiterentwicklung seines Angebots technische Systeme für ein „verursachergerechtes Abfallmanagement“" an, bei dem eine individuelle Erfassung und Abrechnung der Restmüllmengen durch Müllidentsysteme ermöglicht wird. Dabei werden in den Wohnanlagen Müllschleusen installiert, die äußerlich kaum von einer normalen Standardwaschbetonbox zu unterscheiden sind, wo der Müll jedoch nur mit einem Transponderchip eingefüllt werden kann und dabei gewogen wird. Durch die individuelle Zurechnung wird erreicht, dass die Bewohner tatsächlich nur für ihre individuelle Restmüllmenge Abfallgebühren zahlen müssen und somit einen echten Anreiz haben, durch eine optimierte Sortierung und abfallbewussten Konsum Gebühren einzusparen.

Auch wenn der Ansatz auf einer technischen Innovation mit den individuellen Transponderchips basiert, setzt er doch vor allem auf eine Verhaltensänderung der Mieter und kombiniert damit effiziente Technologie mit veränderten Konsummustern zur Nutzenbefriedigung. Schwerpunkt der Dienstleistungen von Innotec ist zwar die Behebung von Fehlbefüllungen direkt am Müllplatz, ein wesentlicher Bestandteil des Konzepts ist nach Angaben von Innotec jedoch auch die Mieterinformation und die Entwicklung eines grundsätzlichen Bewusstseins über Kosten und Einsparpotentiale im Bereich Abfall (Innotec 2004, S. 8). Diese notwendige Wissensvermittlung muss sehr sorgfältig auf die stufenweise Veränderung der Erfassungsinfrastruktur angepasst werden, u. a. müssen die Mieter regelmäßig über Sinn und Erfolg der einzelnen Maßnahmen (mehr Gelbe Tonnen, Einführung der Biotonne, Einführung der Müllschleuse, Begrenzung der Einfüllmengen etc.) informiert werden. Die Einbindung der Mieter in das Konzept ist auch deshalb von zentraler Bedeutung, damit nicht einfach ökonomischen Anreizen folgend der Müll einfach in der Umgebung abgelagert wird, um Kosten zu sparen. Untersuchungen des Witzenhausen Instituts nach Einführung von Müllschleusen haben jedoch gezeigt, dass durch die intensive Beratung sowohl keine Zunahme von wilden Müllablagerungen in der Umgebung festzustellen waren als auch die Sauberkeit an den Müllplätzen selber deutlich erhöht wurde (Witzenhausen-Institut 2007, S. 58). Mittlerweile betreut das Unternehmen bundesweit ca. 250 Wohnungsgesellschaften mit 630.000 Wohneinheiten und 1,5 Mio. Mietern (Innotec 2009). Innotec konzentriert seine Aktivitäten dabei weitestgehend auf den sozialen bzw. öffentlich geförderten Wohnungsbau, wo die Mieter wegen Sprachbarrieren, Bildungsniveau und Zugehörigkeit zu sozialen Schichten in der Regel über kein ausgeprägtes Bewusstsein für die Mülltrennung oder die Vermeidung von Abfällen verfügen. 
Wissenschaftlich begleitete Auswertungen von Innotec- Projekten in Hamburg und Erfurt haben ergeben, dass durch Einführung der verursacher-orientierten Gebührenabrechnung vor allem die Menge der getrennt erfassten Wertstoffe deutlich erhöht wurde und somit mehr Stoffe einem stofflichen Recycling zugeführt werden konnten. Damit konnten die mit den Abfällen verbundenen CO2-Belastungen um bis zu 60 \% gesenkt werden (Hunklinger 2011, S. 17). So ist z. B. in Erfurt die Menge gesammelter Verpackungen aus dem Dualen System um 6o \%, die getrennt erfassten Bioabfälle sogar um $400 \%$ erhöht worden. Das einwohnerspezifische Restmüllaufkommen konnte dadurch von $3,85 \mathrm{~kg}$ pro Woche auf o,8 kg gesenkt werden (Kern 2007, S. 38). Betrachtet man insgesamt alle Verwertungswege wie z. B. Wertstoffhöfe, so ergibt sich eine Rest-Menge von 800 g pro Einwohner und Woche, die entweder tatsächlich vermieden oder verlagert werden, z. B. auf die Müllentsorgung am Arbeitsplatz. Die wissenschaftliche Begleitstudie kommt zu folgendem Fazit: „Die Einführung der Müllschleusen hat die angeschlossenen Bewohner dazu bewegt, die abfallwirtschaftlichen Grundgedanken der Vermeidung und Verwertung in ihrem Entsorgungsverhalten umzusetzen.“ (ebd.).

Das Abfallcontracting hat ihren Business Case durch die Kosteneinsparungen bei der Restmüllentsorgung. Für den Vermieter sind die Kosten insgesamt für die Müllentsorgung in vergangenen Projekten vor allem durch die nachträgliche Müllsortierung abhängig von den abfallwirtschaftlichen Rahmenbedingungen um 20 $\%-50 \%$, in Einzelfällen um bis zu 70 \% gesunken (Innotec 2009, S. 11) - wodurch die Immobilien zu wettbewerbsfähigeren Konditionen am Markt angeboten werden können. Innotec finanziert sich dabei ausschließlich über eine Gewinnbeteiligung an diesen eingesparten Kosten. In der Regel wird in den Projekten eine Pilotphase von fünf Jahren vereinbart, in denen Innotec 75 \% der eingesparten Restmüllgebühren erhält, um die Anlagentechnik und die zu Beginn sehr personalintensive Mieterberatung zu refinanzieren. Nach Ablauf dieser Pilotphase erhält Innotec bei Fortführung des Projekts noch 50 \% der erzielten Einsparungen. Als begünstigender Faktor erweist sich dabei, dass die Aufwendungen des Besitzers für Abfallmanagementdienstleistungen als haushaltsnahe Dienstleistungen im Rahmen der Einkommenssteuer geltend gemacht werden können. Für die Mieter ergeben sich zum einen Vorteile, da die entstehenden Kosten in der Wohnanlage nicht mehr wie bisher pauschal pro Kopf oder Quadratmeter, sondern nach dem individuellen Müllverhalten berechnet werden. Zum anderen führt die optimierte Getrenntsammlung und Behebung von Fehlbefüllungen insgesamt $\mathrm{zu}$ einer deutlichen Reduktion der Restabfallgebühren. Innotec argumentiert, dass auch die Städte durch das optimierte Abfallmanagement profitieren: Da in Großwohnanlagen durchschnittlich ca. 20 \% Sozialhilfe beziehende Transferempfänger wohnen, führen die verminderten Mietkosten zu niedrigeren Mietzuschüssen. Am Beispiel von Duisburg ergäbe sich danach bei 1,7 Mio. $€$ Mietkosteneinsparungen eine kommunale Ersparnis von $180.000 €$ (vgl. Innotec 2011).

\section{Fallbeispiel Repair Café}

Ein weiterer Ansatz für ein suffizienz-orientiertes Geschäftsmodell der Abfallwirtschaft ist das kostenfreie Reparieren von kaputten Gegenständen. Das Geschäftsmodell basiert hierbei unter anderem auf der Erhöhung des Verbraucherbewusstseins für Reparatur - und führt damit schließlich zu einer Ankurbelung der konventionellen Reparaturwirtschaft. Durch die Verlängerung der Nutzungsdauer von Produkten werden Ressourcen für die Produktion von Substitutionsprodukten eingespart, gleichzeitig werden die bisherigen 
Konsumfunktionen jedoch erhalten (Paech 2013). Entstehende Kostenersparnisse resultieren hierbei im Sinne der Suffizienzstrategie nicht in einem weiteren Konsum. Die konkrete Umsetzung des Geschäftsmodells basiert beispielsweise auf Konzepten wie etwa den Repair Cafés, das seinen Ursprung 2009 in den Niederlanden hat. Repair Cafés sind Veranstaltungen, bei denen Reparaturexperten und Verbraucher mit kaputten Gegenständen zusammenkommen und bei Kaffee und Tee diese gemeinsam reparieren. Die Organisation der Veranstaltungen als auch das Engagement der Experten basiert auf ehrenamtlicher Tätigkeit. Egal ob Kleidung, Möbel, elektrische Geräte oder Fahrräder, die Reparaturen sind kostenlos. Jeder kann solche Reparaturtreffen veranstalten und wird von der übergeordneten Organisation „Stichting Repair Cafe““ unterstützt. Europaweit kann gegen einen kleinen Unkostenbeitrag ein Informationspaket und Zusatzmaterialien, wie etwa Vorlagen für Poster und Flyer etc., erworben werden. Im Gegenzug wird die Veranstaltung als offizielles Repair Café auf der Website aufgenommen und erhält dadurch entsprechende öffentliche Aufmerksamkeit.

Während manche Repair-Café-Reparateure handwerkliches Geschick mitbringen und aus Interesse zu richtigen Tüftlern geworden sind, beteiligen sich auch gewerblich tätige Reparatur-Experten an den Veranstaltungen. So ist es beispielsweise im Repair Café in Berlin-Kreuzberg möglich, defekte Smartphones selbständig unter Aufsicht von Technikern der iDoc - 1. Hilfe für Mobiltelefone $\mathrm{GmbH}$ zu reparieren (Kunst-Stoffe o. J.). Seit 2013 stellt das Unternehmen Schrittfür Schritt Reparaturanleitungen kostenlos zur Verfügung und bietet Ersatzteile und Spezialwerkzeug zum Verkauf an, um auf diesem Weg vielen Interessierten die Möglichkeit zu geben die selbständige Reparatur dem Wegwerfen vorzuziehen. Auf den Repair Café Veranstaltungen bietet iDoc die notwendigen Ersatzteile stark vergünstigt an (ca. 50 \% Rabatt) (ebd.).

Mit Blick auf die Senkungspotenziale des Ressourcenverbrauchs durch eine Produktnutzungsdauerverlängerung geraten insbesondere Elektro- und Elektronikgeräte wie etwa Smartphones in den Fokus, denn sie enthalten viele wertvolle Rohstoffe wie Edelmetalle oder Seltene Erden. Rund 30 g Gold werden in einem Smartphone verbaut (Sander et al. 2012). Um $1 \mathrm{~kg}$ Gold herzustellen werden rund 540.000 kg Rohstoffe benötigt (Wuppertal Institut 2014). Insgesamt trägt ein Mobiltelefon etwa einen 75,3 kg schweren ökologischen Rucksack (mehr als 940-mal schwerer als das Mobiltelefon selbst) mit sich herum (Wuppertal Institut 2013). Bedenkt man, dass 2013 rund 93 \% der deutschen Haushalte mit Mobiltelefonen ausgestattet waren (Destatis 2015), wird das hohe Ressourceneinparpotenzial durch eine längere Nutzung der Geräte deutlich. Insbesondere, wenn man berücksichtigt, dass derzeit die realen Nutzungsdauern und technischen Lebensdauern speziell von Elektro- und Elektronikgeräten oftmals weit auseinander gehen. So liegt beispielsweise die durchschnittliche Nutzungsdauer von Smartphones bei etwa 2 Jahren (Manhart et al. 2012), obwohl diese oftmals noch funktionsfähig sind. Aus ressourcentechnischer Sicht steigen die Ressourceneinsparpotenziale einer Produktnutzungsdauerverlängerung mit zunehmender Diskrepanz zwischen realer Nutzungsdauer und technischer Lebensdauer an. Aber nicht nur mit einer längeren Nutzung von Elektro- und Elektronikgeräten sondern auch mit der Reparatur von Kleidung und Möbeln, bis hin zu Fahrrädern, wird materielle Produktion subsituiert und dadurch wertvolle Ressourcen eingespart. Bei den Repair Cafés werden etwa 70 \% der Geräte und Produkte, die von den Besuchern der Repair Cafés mitgebracht werden, auch tatsächlich repariert (Wilts und von Gries 2013). 
Hinsichtlich des Buisness Case profitiert die Reparaturbranche vor allem von dem höheren Verbraucherbewusstsein für Reparatur, dass durch die Repair Cafés in die Gesellschaft getragen wird. Nehmen speziell gewerblich tätige Reparatur-Experten an lokalen Repair Cafés teil, rechnet sich die Teilnahme zusätzlich durch den Aufbau von Kundenkontakten, wodurch auch Vorteile der Mundpropaganda nutzbar werden. Auch die konkreten Hilfestellungen bei der Reparatur sind eine wichtige Referenz, die eigene Folgeleistungen besser verkaufen lassen. Gleichzeitig können durch den Verkauf von Ersatzteilen auf den Veranstaltungen, wie es etwa iDoc praktiziert, Einnahmen direkt generiert werden. Für den Verbraucher können sich durch die Reparatur anstelle des Neukaufs Kosteneinsparungen ergeben. Durch den direkten Kontakt mit Experten konventioneller Reparatur-Betriebe erhalten die Verbraucher wertvolle Informationen und Möglichkeiten lokaler Reparaturdienste. Bei weiteren Reparaturen (die nicht im Rahmen der Repair Cafés durchgeführt werden, z. B. aufgrund von Komplextät, fehlender Ersatzteile, Zeitmangel etc.) verringern sich dadurch die Transaktionskosten der Reparatur, die oftmals höher als beim Neukauf sind (z. B. durch Möglichkeiten des Erwerbs von Neuprodukten im Internet mit wenigen klicken und kostenloser Lieferung am nächsten Tag). Zu dem wird dem Verbraucher durch die Teilnahme an den Repair Cafés bewusst, das Reparatur und Qualität durchaus korrelieren. Die Stärkung des Bewusstseins für Reparatur im Sinne des Umweltschutzes und der Unterstützung lokaler Betriebe führt dazu, dass bei der Entscheidungsabwägung Reparatur oder Neukauf nicht die Kosten allein berücksichtigt werden (González 2013). Hierdurch wird angestoßen, dass auch bei Produkten mit abnehmenden Preisgefälle zwischen Reparatur und Neukauf dennoch die Reparatur eine attraktive Alternative darstellen kann.

\section{Schlussfolgerungen}

Beide Fallbeispiele verdeutlichen die Potentiale, die sich durch den Funktionswandel der Abfallwirtschaft - weg von der Gewährleistung einer Entsorgungssicherheit hin zu einem nachhaltigen Ressourcenmanagement - in Form suffizienzorientierter Geschäftsmodelle ergeben können. Wo Abfälle als Materialisierung verschwendeter Ressourcen nicht nur mit volkswirtschaftlichen Kosten, sondern auch konkreten Abfallgebühren etc. für Unternehmen und Haushalten verbunden sind, bieten Suffizienz-Ansätze im Sinne eines „Weniger“ Möglichkeiten zur Steigerung der Ressourceneffizienz bei gleichzeitigen positiven sozio-ökonomischen Effekten. Gleichzeitig weisen sie auch auf grundlegende Herausforderungen hin, die ihrer konkreten Umsetzung über einzelne Innovationsnischen hinaus entgegenstehen:

- In der Diskussion um die Entwicklung zu einer Recyclinggesellschaft oder aktuell um das durch die neue Europäische Kommission zurückgenommene „Circular Economy Package“ wird regelmäßig auf die mit Konsistenz- und Effizienzansätzen verbundenen Ressourceneinspar- und Beschäftigungspotenziale hingewiesen (Bastein et al. 2013; EMF 2013) - für Suffizienzstrategien der Abfallvermeidung fehlen bisher weitestgehend Konzepte, entsprechende positive Effekte in quantitativen Größen auszudrücken: Wie misst man Abfall, der nicht entstanden ist? Auch die Effekte einer verlängerten Produktnutzungsdauer gestalten sich im Vergleich zu technischen Recyclingprozessen äußerst komplex, da sie im hohen Maße von individuellen Nutzungsmustern abhängen - beispielsweise werden viele reparierte als zusätzliche Zweitgeräte betrieben, so dass im Endeffekt eher mehr Energie für die Nutzung aufgewendet werden muss und letztendlich mehr Abfälle entstehen. Die Entwicklung entsprechender Indikatoren, die 
solche sozio-ökonomischen Aspekte berücksichtigen könnten, steckt weitestgehend noch in den Kinderschuhen.

- Die massiven Pfadabhängigkeiten technischer Infrastruktursysteme stehen der Diffusion von Suffizienz-Innovationen wie Repair Cafés oder AbfallContracting-Modelle entgegen: Das über Jahrzehnte hinweg optimierte System der Abfallsammlung und -verwertung ist tief verwurzelt in Konsummustern, Rechtsvorschriften oder ökonomischen Interessen insbesondere private und öffentliche Milliarden-Investitionen in technische Infrastrukturen der Abfallbehandlung wie z. B. Müllverbrennungsanlagen rechnen sich nur bei einem entsprechenden Abfallaufkommen und setzen damit klare Anreize, eine konsequente Ausrichtung auf die Vermeidung von Abfällen nur sehr zögerlich umzusetzen.

Während abfallwirtschaftliche Effizienz- und Konsistenzansätze in ihren Auswirkungen durch etablierte Konzepte der Technikfolgenabschätzung oder Marktpotentialanalysen zumindest größenordnungsmäßig kalkulierbar sind, stellen die dargestellten Suffizienzkonzepte durch ihre hohe Innovationsdynamik und die komplexe Interaktion von Produzenten und Konsumenten die etablierte Infrastrukturplanung vor enorme Herausforderungen: Mit dem Ausmaß der Unsicherheiten über zukünftige Einspareffekte steigt damit der Anreiz, vorzugsweise auf inkrementelle, aber kalkulierbare Innovationen zu setzen. Die Entwicklung modularer und flexibler Anlagenkonzepte und die Abkehr von TopdownPlanungsprozessen hin zu einer bedürfnisorientierten Infrastrukturplanung könnend damit als zentrale Herausforderung für die zukünftige Entwicklung der hier skizzierten Suffizienz-basierten Geschäftsmodelle in der Abfallwirtschaft gelten - ein bisher vor allem in Deutschland kaum adressiertes Forschungsfeld für interdisziplinäre und transformative Forschung.

\section{Literatur}

Bastein T, Roelofs E, Rietveld E, Hoogendoorn A (2013) Opportunities for a circular economy in The Netherlands. ISBN 97890-6986-436-8

Bringezu S, Bleischwitz R (2009) Sustainable resource management. Trends, visions and policies for Europe and the World. Greenleaf Publisher, Sheffield

Destatis (2015) Staat \& Gesellschaft - Ausstattung mit Gebrauchsgütern Ausstattung privater Haushalte mit Informations- und Kommunikationstechnik $\begin{array}{llll}\text { Deutschland } & - & \text { Statistisches } & \text { Bundesamt (Destatis). }\end{array}$ https://www.destatis.de/DE/ZahlenFakten/

GesellschaftStaat/EinkommenKonsumLebensbedingungen/AusstattungGebrauchsgu etern/Tabellen/Infotechnik_D.html.ZZugegriffen: 16. Feb. 2015

EMF (2013) Towards the circular Economy: opportunities for the consumer goods sector. Report 2. http://www.ellenmacarthurfoundation.org/business/reports/ce2013. Zugegriffen: 17. Feb. 2015

Fischer S, O'Brien M, Wilts H, Steger S, Schepelmann P, Jordan ND, Rademacher B (2015) Waste Prevention in a "Leasing Society“. Int J Waste Resour 5:170. doi:10.4172/2252-5211.1000170 
González JG (2013) Vorbereitung zur Wiederverwendung: Regelung und Regelungsbedarf-Umsetzungs-und Erfolgsaussichten. Leuphana Schriftenreihe Nachhaltigkeit \& Recht, http://www.leuphana.de/fileadmin/user_upload/Forschungseinrichtungen/profess uren/energie-und-umweltrecht/Schriftenreihe/NR_-

Nr._3_Guerra_WV_def.pdf. Zugegriffen: 17. Feb. 2015

Hemmer D, Höferl A, Hollos B (2003) Privatisierung und Liberalisierung öffentlicher Dienstleistungen in der EU-15: Abfallwirtschaft. Studie der Österreichischen Gesellschaft für Politikberatung und Politikentwicklung, Wien

Hunklinger R (2011) Die intelligente Tonne. Innotec bietet modernes Abfallmanagement: Mülltonnen, deren Chip die Einwürfe zählt. Cleantech magazin 1/2011:16-17

Innotec Abfallmanagement GmbH (2004) GWH setzt auf Abfallmanagement. Pilotprojekt in Bad Vilbel. Pressebericht vom 06.04.2004. http://www.innotecgruppe.de/docs/04_geschaeftsbericht_gwh04.pdf. Zugegriffen: 01. Dez. 2011

Innotec Abfallmanagement GmbH (2009) Der Innotec-Quotient. Modernes Abfallmanagement. Broschüre. http://www.innotecabfallmanagement.de/docs/innotecgruppe_imagebroschuere.pdf .Zugegriffen: 01. Dez. 2011

Innotec Abfallmanagement GmbH (2011) Was leisten private Anbieter im Entsorgungs-Standortservice? Pressemitteilung vom 11.1.2011. http://www.recyclingportal.eu/artikel/25861.shtml. Zugegriffen: 11. Jan. 2011

Innotec Abfallmanagement GmbH (o. J.) Konventionelles Abfallmanagement. Kiel. http://www.innotec-abfallmanagement.de/de/konventionellesabfallmanagement.htm. Zugegriffen: 01. Dez. 2011

Kern M (2007) Ergebnispräsentation von verursachergerechten Abfallmanagementsystemen im Wohnungsbau am Beispiel Müllschleusen in Erfurt. Praxisforum 20.11.2007, Witzenhausen. http://www.witzenhauseninstitut.de/downloads/Praxisforum_2007_Kern.pdf. Zugegriffen: 01. Dez. 2011

Kuchenbuch L (1989) Abfall. Eine Stichwortgeschichte. In: Hoffmann O (Hrsg) Ex und Hopp. Das Prinzip Wegwerf. Eine Bilanz mit Verlusten. Katalog zur Ausstellung in Frankfurt im Auftrag des Deutschen Werkbundes. Frankfurt a. M., S 33-37

Kunst-Stoffe (o. J.) Repair cafe. https://www.kunst-stoffeberlin.de/index.php?option=com_content\&view $=$ article\&id=267:repaircafe\&catid $=5$ 3:repair-cafe\&Itemid=179\&lang=en. Zugegriffen: 16. Feb. 2015

Lamping W (1998) Kommunale Abfallpolitik. Ökologischer Strukturwandel und politisches Lernen. Dissertationsschrift an der Fakultät für Geistes- und Sozialwissenschaften der Universität Hannover, Hannover

Linz M (2012) Weder Mangel noch Übermaß: Warum Suffizienz unentbehrlich ist. Oekom Verlag, München

Linz M, Bartelmus P, Hennicke P, Jungkeit R, Sachs W, Scherhorn G, Wilke G, von 
Winterfeld U (2002) Von nichts zuviel. Suffizienz gehört zur Zukunftsfähigkeit. Über ein Arbeitsvorhaben des Wuppertal Instituts.; Wuppertal Institut für Klima, Umwelt, Energie 2002

Manhart A, Riewe T, Brommer E (2012) PROSA Smartphones. Entwicklung der Vergabekriterien für ein klimaschutzbezogenes Umweltzeichen. Studie im Rahmen des Projekts „Top 100- Umweltzeichen für klima- relevante Produkte“. August 2012, Freiburg

McDonough W, Braungart M (2010) Cradle to cradle: remaking the way we make things. MacMillan. North Point Press, New York

Paech N (2012) Befreiung vom Überfluss. Auf dem Weg in die Postwachstumsökonomie. Oekom Verlag, München

Paech N (2013) Weniger und einfacher. Jenseits der Wachstumsspirale. Forschung \& Lehre 4:276-277

Sachs W (1993) Die vier E's. Merkposten für einen maßvollen Wirtschaftsstil. Politische Ökologie, Jg. 11, Nr. 33, S. 69-72

Sachs W (2002) Die zwei Gesichter der Ressourcenproduktivität. In: Linz M (Hrsg) Von nichts zu viel - Suffizienz gehört zur Zukunftsfähigkeit. Wuppertal Papers Nr. 125, Wuppertal, S. 49-56

Sander K, Schilling S, Marscheider-Weidemann F, Wilts H, von Gries N, Hobohm J (2012) Abfallwirtschaftliche Produktverantwortung unter Ressourcenschutzaspekten. Meilensteinbericht. UFOPLAN FKZ 371195318

Schenkel W (2003) Zur Geschichte der Abfallwirtschaft in Deutschland. Müll und Abfall 35(12):620-625

Scherhorn G (2002) Die Logik der Suffizienz. In Linz M (Hrsg) Von nichts zu viel Suffizienz gehört zur Zukunftsfähigkeit. Wuppertal Papers Nr. 125, Wuppertal, S. 1526

Schneidewind U, Palzkill A (2012) Suffizienz als Business Case. Nachhaltiges Ressourcenmanagement als Gegenstand einer transdisziplinären Betriebswirtschaftslehre. Impulse zur WachstumsWende 2: Wuppertal Institut für Klima Umwelt, Energie. Wuppertal

Sommer A. (2012) ,Managing Green Business Model Transformations.' Dissertation, Springer Verlag

Stengel O (2011) Suffizienz: Die Konsumgesellschaft in der ökologischen Krise. Oekom Verlag, München

Wilts H, Palzkill A (2014) Suffizienz als Geschäftsmodell: Contracting. Kurzanalyse AP2 im Projekt Ressourcenpolitik: Analyse der ressourcenpolitischen Debatte und Entwicklung von Politikoptionen (PolRess)

Wilts H, Schepelmann P (2015) Waste Policy Brief. Deliverable 11.1, FP 7 
Environmental Macro Indicators for Innovation Project (EMInInn). Im Erscheinen, Wuppertal

Wilts H, von Gries N (2013) „Reuse - One Step Beyond.“ Machbarkeitsstudie, Wuppertal

Witzenhausen-Institut (2007) Wissenschaftliche Begleitung der Einführung von Müllschleusen in der Stadt Erfurt. Abschlussbericht, Witzenhausen

Wollny V (1992) Abschied vom Müll. Perspektiven für Abfallvermeidung und eine ökologische Stoffflußwirtschaft. Studie des Öko- Instituts, Göttingen

Wuppertal Institut (2013) 18 Factsheets zum Thema Mobiltelefone und Nachhaltigkeit.

http://wupperinst.org/uploads/tx_wupperinst/Mobiltelefone_Factsheets.pdf.

Zugegriffen: 17. Feb. 2015

Wuppertal Institut (2014) Materialintensität von Materialien, Energieträgern, Transportleistungen, Lebensmittel. http://wupperinst.org/ uploads/tx_wupperinst/MIT_2014.pdf. Zugegriffen: 17. Feb. 2015 